\title{
TORSION OF ELLIPTIC CURVES WITH RATIONAL $j$-INVARIANT DEFINED OVER NUMBER FIELDS OF PRIME DEGREE
}

\author{
TOMISLAV GUŽVIĆ
}

\begin{abstract}
Let $[K: \mathbb{Q}]=p$ be a prime number and let $E / K$ be an elliptic curve with $j(E) \in \mathbb{Q}$. We determine the all possibilities for $E(K)_{\text {tors. }}$. We obtain these results by studying Galois representations of $E$ and of it's quadratic twists.
\end{abstract}

\section{INTRODUCTION}

Let $K$ be a number field such that $[K: \mathbb{Q}]=d$ and let $E / K$ be an elliptic curve. The set of $K$-rational points, $E(K)$ can be given a group structure. A theorem of Mazur shows that $E(K)$ is a finitely generated abelian group. Therefore this group can be decomposed as $E(K)=E(K)_{\text {tors }} \oplus \mathbb{Z}^{r}, r \geq 0$. It is known that $E(K)_{\text {tors }}$ is of the form $C_{m} \oplus C_{n}$ for two positive integers $m, n$ such that $m$ divides $n$, where $C_{m}$ and $C_{n}$ denote cyclic groups of order $m$ and $n$, respectively.

One of the goals in the theory of elliptic curves is the classification of torsion groups of elliptic curves defined over various fields. We will now briefly describe results related to this paper.

Let $d$ be a positive integer.

(1) Let $\Phi(d)$ be the set of possible isomorphism classes of groups $E(K)_{\text {tors }}$, where $K$ runs through all number fields $K$ of degree $d$ and $E$ runs through all elliptic curves over $K$. In [31], Merel proved that $\Phi(d)$ is finite for all positive integers $d$. The set $\Phi(1)$ was determined by Mazur ([29]) and $\Phi(2)$ was determined by Kenku, Momose and Kamienny ([21, [20]). Derickx, Etropolski, Hoeij, Morrow and Zureick-Brown have determined $\Phi(3)$ but the result is (at the time of writing this paper) unpublished.

(2) Let $\Phi^{\mathrm{CM}}(d)$ be the set of possible isomorphism classes of groups $E(K)_{\text {tors }}$, where $K$ runs through all number fields $K$ of degree $d$ and $E$ runs through all elliptic curves with $\mathrm{CM}$ over $K . \Phi^{\mathrm{CM}}(1)$ has been determined by Olson in 35 and $\Phi^{\mathrm{CM}}(d)$ for $d=3,4$ by Zimmer and his collaborators. The sets $\Phi^{\mathrm{CM}}(d)$, for $4 \leq d \leq 13$ have been determined by Clark, Corn, Rice and Stankiewicz in [5]. Bourdon and Pollack have determined torsion groups of CM elliptic curves over odd degree number fields in [2].

The author gratefully acknowledges support from the QuantiXLie Center of Excellence, a project co-financed by the Croatian Government and European Union through the European Regional Development Fund - the Competitiveness and Cohesion Operational Programme (Grant KK.01.1.1.01.0004) and by the Croatian Science Foundation under the project no. IP-2018-011313. 
(3) Let $\Phi_{\mathbb{Q}}(d) \subseteq \Phi(d)$ be the set of possible isomorphism classes of groups $E(K)_{\text {tors }}$, where $K$ runs through all number fields $K$ of degree $d$ and $E$ runs through all elliptic curves defined over $\mathbb{Q}$. For $d=2,3$, the sets $\Phi_{\mathbb{Q}}(d)$ have been determined by Najman $\left([33)\right.$ while $\Phi_{\mathbb{Q}}(4)$ has been determined by Chou (44) and González-Jiménez, Najman (13]). The set $\Phi_{\mathbb{Q}}(5)$ has been determined by González-Jiménez in [10. González-Jiménez and Najman have also proved that $\Phi_{\mathbb{Q}}(p)=\Phi(1)$ for prime $p \geq 7$ in [13]. In [15], the author has partially determined $\Phi_{\mathbb{Q}}(6)$.

(4) Let $\Phi_{j \in \mathbb{Q}}(d)$ be the set of possible isomorphism classes of groups $E(K)_{\text {tors }}$, where $K$ runs through all number fields $K$ of degree $d$ and $E$ runs through all elliptic curves defined over $K$ such that $j(E) \in \mathbb{Q}$.

(5) Let $R_{\mathbb{Q}}(d)$ be the set of all primes $p$ such that there exists a number field $K$ of degree $d$, an elliptic curve $E / \mathbb{Q}$ such that there exists a point of order $p$ on $E(K)$. This set has been determined by González-Jiménez and Najman $([13)$.

We now state the main results of this paper in which we classify the sets $\Phi_{j \in \mathbb{Q}}(p)$, where $p$ is a prime number.

Theorem 1.1. Let $K$ be a number field such that $[K: \mathbb{Q}]=p \geq 7$ is prime and let $E / K$ be an elliptic curve with rational $j$-invariant. If $E(K)$ contains a point of order $n>1$, then $C_{n} \in \Phi(1)$.

Theorem 1.2. Let $K$ be a number field such that $[K: \mathbb{Q}]=5$ and let $E / K$ be an elliptic curve with rational $j$-invariant. If $E(K)$ contains a point of order $n>1$, then $C_{n} \in \Phi_{\mathbb{Q}}(5)$.

Theorem 1.3. Let $K$ be a number field such that $[K: \mathbb{Q}]=3$ and let $E / K$ be an elliptic curve with rational $j$-invariant. If $E(K)$ contains a point of order $n>1$, then $C_{n} \in \Phi_{\mathbb{Q}}(3)$.

Theorem 1.4. Let $K$ be a number field such that $[K: \mathbb{Q}]=2$ and let $E / K$ be an elliptic curve with rational $j$-invariant. If $E(K)$ contains a point of order $n>1$, then $C_{n} \in \Phi_{\mathbb{Q}}(2) \cup\left\{C_{13}\right\}$.

Obviously we have $\Phi_{\mathbb{Q}}(d) \subseteq \Phi_{j \in \mathbb{Q}}(d)$. If $E / K$ is an elliptic curve such that $j(E) \in \mathbb{Q} \backslash\{0,1728\}$, take $E^{\prime} / \mathbb{Q}$ to be any elliptic curve such that $j\left(E^{\prime}\right)=j(E)$. Then $E$ and $E^{\prime}$ are either isomorphic over $K$ or over some quadratic extension $L$ of $K$. Assume that $C_{m} \oplus C_{n} \subseteq E(K)$. This implies that $C_{m} \oplus C_{n} \subseteq E^{\prime}(L)$, so $C_{m} \oplus C_{n}$ is a subgroup of one of the groups appearing in $\Phi_{\mathbb{Q}}(2 d)$.

Let us consider the case when $d$ is odd. Assume that $C_{m} \oplus C_{n}$, where $m$ divides $n$ is contained in $E(K)$. By the properties of Weil pairing we have $\mathbb{Q}\left(\zeta_{m}\right) \subseteq K$. If $m \geq 3,\left[\mathbb{Q}\left(\zeta_{m}\right): \mathbb{Q}\right]$ is even so $\mathbb{Q}\left(\zeta_{m}\right)$ can't be a subfield of $K$. Therefore, when trying to classify $\Phi_{j \in \mathbb{Q}}(p)$ we shall consider only groups of the form $C_{n}$ and $C_{2} \oplus C_{2 n}$.

We now describe the general strategy used to solve this problem. Let $K$ be a number field of degree $p$ and $E(K)$ be an elliptic curve with $j(E) \in \mathbb{Q} \backslash\{0,1728\}$ and let $P_{n} \in E(K)$ be a point of order $n$. If $E$ is a base change of an elliptic curve defined over $\mathbb{Q}$, we are done, because $E(K)_{\text {tors }} \in \Phi_{\mathbb{Q}}(p)$. Otherwise, we take any elliptic curve $E^{\prime}$ defined over $\mathbb{Q}$ such that $j\left(E^{\prime}\right)=j(E)$. With $L$ we shall denote 
(unless otherwise stated) a quadratic extension of $K$ such that $E$ and $E^{\prime}$ are isomorphic over $L$, so they are quadratic twists of each other. On the one hand, if $\left\{P_{n}, Q_{n}\right\}$ is a basis for $E[n]$, the image of $\rho_{E, n}$ is a subgroup of $B_{0}$, where

$$
B_{0}:=\left\{\left[\begin{array}{rr}
1 & * \\
0 & *
\end{array}\right]\right\} .
$$

Since $\rho_{E, n} \sim \chi \cdot \rho_{E^{\prime}, n}$, where $\chi$ is a quadratic character, we can obtain some information about the $\bmod n$ Galois representation of $E^{\prime}$. On the other hand, since $E$ and $E^{\prime}$ are isomorphic over $L$, there exists a point $P_{n}^{\prime} \in E^{\prime}(L)$ of order $n$. For each prime divisor $q$ of $n$, let $P_{q}^{\prime} \in E^{\prime}(L)$ be a point of order $q$. Obviously $\left[\mathbb{Q}\left(P_{q}^{\prime}\right): \mathbb{Q}\right]$ is a divisor of $[L: \mathbb{Q}]$. Using the results of $[13$, we can check the possible values of $\left[\mathbb{Q}\left(P_{q}^{\prime}\right): \mathbb{Q}\right]$. Often it turns out that $E^{\prime}$ has a rational $q$-isogeny.

\section{NotATION AND AUXILIARY RESULts}

Let $E / F$ be an elliptic curve defined over a number field $F$. There exists an $F$-rational cyclic isogeny $\phi: E \rightarrow E^{\prime}$ of degree $n$ if and only if $\operatorname{ker} \phi$ is a $\operatorname{Gal}(\bar{F} / F)$ invariant cyclic group of order $n$; in this case we say that $E$ has an $F$-rational $n$-isogeny. When $F=\mathbb{Q}$, possible degrees $n$ of elliptic curves over $\mathbb{Q}$ are known by the following theorem.

Theorem 2.1 (Mazur, 29]). Let $E / \mathbb{Q}$ be an elliptic curve. Then

$$
E(\mathbb{Q})_{t o r s} \cong \begin{cases}C_{m}, & m=1, \ldots, 10,12, \\ C_{2} \oplus C_{2 m}, & m=1, \ldots, 4 .\end{cases}
$$

Theorem 2.2 (Kenku, Momose, [21], Kamienny [20]). Let $E / F$ be an elliptic curve over a quadratic number field $F$. Then

$$
E(F)_{\text {tors }} \cong \begin{cases}C_{m}, & m=1, \ldots, 16,18, \\ C_{2} \oplus C_{2 m}, & m=1, \ldots, 6, \\ C_{3} \oplus C_{3 m}, & m=1,2, \\ C_{4} \oplus C_{4} . & \end{cases}
$$

Theorem 2.3 (Mazur [30], Kenku 22], 23], 24], 25]). Let $E / \mathbb{Q}$ be an elliptic curve with a rational $n$-isogeny. Then

$$
n \in\{1, \ldots, 19,21,25,27,37,43,67,163\} .
$$

There are infinitely many elliptic curves (up to $\overline{\mathbb{Q}}$-isomorphism) with a rational $n$-isogeny over $\mathbb{Q}$ for

$$
n \in\{1, \ldots, 10,12,13,16,18,25\}
$$

and only finitely many for all the other $n$.

Theorem 2.4. [33, Theorem 2] Let $E / \mathbb{Q}$ be an elliptic curve and $F$ a quadratic field. Then

$$
E(F)_{\text {tors }} \cong \begin{cases}C_{m}, & m=1, \ldots, 10,12,15,16 \\ C_{2} \oplus C_{2 m}, & m=1, \ldots, 6 \\ C_{3} \oplus C_{3 m}, & m=1,2 \\ C_{4} \oplus C_{4} . & \end{cases}
$$


Theorem 2.5. 33, Theorem 1] Let $E / \mathbb{Q}$ be an elliptic curve and $K$ a cubic field. Then

$$
E(K)_{\text {tors }} \cong \begin{cases}C_{m}, & m=1, \ldots, 10,12,13,14,18,21, \\ C_{2} \oplus C_{2 m}, & m=1, \ldots, 4,7 .\end{cases}
$$

Theorem 2.6. 13, 4, Let $E / \mathbb{Q}$ be an elliptic curve and $K$ a quartic field. Then

$$
E(K)_{\text {tors }} \cong \begin{cases}C_{m}, & m=1, \ldots, 10,12,13,15,16,20,24, \\ C_{2} \oplus C_{2 m}, & m=1, \ldots, 6,8, \\ C_{3} \oplus C_{3 m}, & m=1,2, \\ C_{4} \oplus C_{4 m}, & m=1,2, \\ C_{5} \oplus C_{5}, & \\ C_{6} \oplus C_{6} . & \end{cases}
$$

Theorem 2.7. 9] Let $E / \mathbb{Q}$ be an elliptic curve and $K$ a quintic field. Then

$$
E(K)_{\text {tors }} \cong \begin{cases}C_{m}, & m=1, \ldots, 12,25, \\ C_{2} \oplus C_{2 m}, & m=1, \ldots, 4 .\end{cases}
$$

Theorem 2.8. 15 Let $E / \mathbb{Q}$ be an elliptic curve and let $K$ be a sextic number field. Then

$$
E(K)_{\text {tors }} \cong \begin{cases}C_{m}, & m=1, \ldots, 16,18,21,30, m \neq 11, \\ C_{2} \oplus C_{2 m}, & m=1, \ldots, 7,9, \\ C_{3} \oplus C_{3 m}, & m=1, \ldots, 4, \\ C_{4} \oplus C_{4 m}, & m=1,3, \\ C_{6} \oplus C_{6}, & \\ C_{3} \oplus C_{18} . & \end{cases}
$$

Additionally, if $G_{E}(2) \neq 2 B$, then $E(K)$ is never isomorphic to $C_{3} \oplus C_{18}$.

Galois representations attached to elliptic curves. Let $E / \mathbb{Q}$ be an elliptic curve and let $n$ a positive integer. By $E[n]$ we shall denote the $n$-torsion subgroup of $E(\overline{\mathbb{Q}})$. The field $\mathbb{Q}(E[n])$ is the number field obtained by adjoining to $\mathbb{Q}$ all the $x$ and $y$-coordinates of the points of $E[n]$. The absolute Galois group $\operatorname{Gal}(\overline{\mathbb{Q}} / \mathbb{Q})$ acts on $E[n]$ by its action on the coordinates of the points, inducing a mod $n$ Galois representation attached to $E$ :

$$
\rho_{E, n}: \operatorname{Gal}(\overline{\mathbb{Q}} / \mathbb{Q}) \rightarrow \operatorname{Aut}(E[n]) .
$$

After we fix a base for the $n$-torsion, we can identify $\operatorname{Aut}(E[n])$ with $\mathrm{GL}_{2}(\mathbb{Z} / n \mathbb{Z})$. This means that we can consider $\rho_{E, n}(\mathrm{Gal}(\overline{\mathbb{Q}} / \mathbb{Q}))$ as a subgroup of $\mathrm{GL}_{2}(\mathbb{Z} / n \mathbb{Z})$, uniquely determined up to conjugacy. We shall denote $\rho_{E, n}(\mathrm{Gal}(\overline{\mathbb{Q}} / \mathbb{Q}))$ by $G_{E}(n)$. Moreover, since $\mathbb{Q}(E[n])$ is a Galois extension of $\mathbb{Q}$ and $\operatorname{ker} \rho_{E, n}=\operatorname{Gal}(\overline{\mathbb{Q}} / \mathbb{Q}(E[n]))$, by the first isomorphism theorem we have $G_{E}(n) \cong \operatorname{Gal}(\mathbb{Q}(E[n]) / \mathbb{Q})$.

Rouse and Zureick-Brown [36] have classified all the possible 2-adic images of $\rho_{E, 2^{\infty}}: \operatorname{Gal}(\overline{\mathbb{Q}} / \mathbb{Q}) \rightarrow \mathrm{GL}_{2}\left(\mathbb{Z}_{2}\right)$, and have given explicitly all the 1208 possibe images. We will use the same notation as in [36] for the 2-adic image of a given elliptic curve $E / \mathbb{Q}$. In 27, González-Jiménez and Lozano-Robledo have determined for each possible image the degree of the field of definition of any 2 -subgroup. From 
the results of [11] one can see if a given 2-subgroup is defined over a number field of given degree $d$.

Division polynomial method. $E / \mathbb{Q}$ be an elliptic curve and $n$ a positive integer. We denote by $\psi_{E, n}$ the $n$-th division polynomial of $E$ (see [38, Section 3.2]). If $n$ is odd, then the roots of $\psi_{E, n}$ are precisely the $x$-coordinates of points $P \in E[n]$. Similarly, if $n$ is even, then the roots of $\psi_{E, n} / \psi_{E, 2}$ are precisely the $x$-coordinates of points $P \in E[n] \backslash E[2]$. Let $f_{E, n}$ denote the corresponding primitive $n$-division polynomial associated to $E$, i.e. it's roots are the $x$-coordinates of points $P$ on $E(\overline{\mathbb{Q}})$ of exact order $n$. We briefly describe the construction of $f_{E, n}$. If $n=p$ is prime, then $f_{E, p}=\psi_{E, p}$. For an arbitrary $n$, we have

$$
f_{E, n}:=\frac{\psi_{E, n}}{\prod_{d \mid n, d \neq n} f_{E, d}} .
$$

Note that if $E^{d} / \mathbb{Q}$ is a quadratic twist of $E / \mathbb{Q}$, then $\psi_{E, n}=\alpha \psi_{E^{d}, n}$ and $f_{E, n}=$ $\beta f_{E^{d}, n}$, for some rational constants $\alpha, \beta$. Consider the following problem:

Given a rational number $j$ and $K$ a number field of degree $d$, does there exist an elliptic curve $E / \mathbb{Q}$ such that $j=j(E)$ can $E(K)$ contains a point $P$ of exact order $n$ ?

Let $E_{0} / \mathbb{Q}$ be any elliptic curve with $j=j\left(E_{0}\right)$. In Magma 1 , we compute the primitive division polynomial $f_{E_{0}, n}$. Since every elliptic curve $E / \mathbb{Q}$ with $j(E)=j$ is a quadratic twist of $E_{0}$, we have $f_{E_{0}, n}=\beta f_{E, n}$, for some rational number $\beta$. Next, we factor $f_{E_{0}, n}$ over $\mathbb{Q}[x]$. Let $d^{\prime}$ denote the degree of the smallest irreducible factor $f$ of $f_{E_{0}, n}$ and let $x_{0}$ be a root of $f$. If $d^{\prime}>d$, then $[\mathbb{Q}(P): \mathbb{Q}] \geq\left[\mathbb{Q}\left(x_{0}\right): \mathbb{Q}\right]=d^{\prime}>d=[K: \mathbb{Q}]$ and so a point $P$ of exact order $n$ on $E(\overline{\mathbb{Q}})$ can't be defined over $K$.

Specific elliptic curves mentioned in this paper will be referred to by their LMFDB label and a link to the corresponding LMFDB page 26] will be included for the ease of the reader. Conjugacy classes of subgroups of $\mathrm{GL}_{2}(\mathbb{Z} / p \mathbb{Z})$ will be referred to by the labels introduced by Sutherland in [37.

\section{Classification of $\Phi_{j \in \mathbb{Q}}(p)$}

Lemma 3.1. Let $p \geq 7$ be a prime number. Then $R_{\mathbb{Q}}(2 p)=\{2,3,5,7\}$. Moreover, we have $R_{\mathbb{Q}}(10)=\{2,3,5,7,11\}$ and $R_{\mathbb{Q}}(6)=\{2,3,5,7,13\}$.

Proof. The claim will follow easily by [13, Corollary 6.1.]. We briefly sketch the proof. Let $p \geq 7$ and $q \geq 23, q \neq 37,43,67,163$ be a prime numbers and assume that $q \in R_{\mathbb{Q}}(2 p)$. We have that $2(q-1) \mid 2 p$ or $\frac{q^{2}-1}{3} \mid 2 p$. If $2(q-1) \mid 2 p$, we have $q \in\{2, p+1\}$, which is impossible. If $\frac{q^{2}-1}{3} \mid 2 p$, it follows that $(q-1)(q+1) \mid 6 p$. If $q-1 \geq p$, then $(q-1)(q+1)>p^{2}>6 p$, a contradiction. Therefore $q-1 \in\{1,2,3,6\}$, so $q \leq 7$ which is impossible since $q \geq 23$. It remains to check that the claim holds for $q \in\{11,13,17,19,37,43,67,163\}$ which is trivial to do.

The claims $R_{\mathbb{Q}}(10)=\{2,3,5,7,11\}$ and $R_{\mathbb{Q}}(6)=\{2,3,5,7,13\}$ also easily follow from [13, Corollary 6.1.]. 
Theorem 3.2. Let $K$ be a number field of prime degree $p$ and let $E / K$ be a $\mathrm{CM}$ elliptic curve with $j(E) \in \mathbb{Q}$. Then we have $E(K)_{\text {tors }} \in \Phi_{\mathbb{Q}}(p)$.

Proof. The claim follows easily by [2, Theorem 1.2.], [3, Theorem 1.5.] and Lemma 3.1.

Therefore, from now on we shall assume that elliptic curves we're dealing with do not have CM.

Lemma 3.3. Let $E / \mathbb{Q}$ be an elliptic curve and $p \geq 3$ a prime and $[F: \mathbb{Q}]=2$. Assume that $E(F)[p] \supseteq C_{p}$, but $E(\mathbb{Q})[p]=O$. Then there there exists quadratic twist $E^{\prime} / \mathbb{Q}$ of $E / \mathbb{Q}$ such that $E^{\prime}(\mathbb{Q})[p]=C_{p}$.

Proof. Since $F=\mathbb{Q}(\sqrt{d})$, put $E^{\prime}:=(E)^{d}$. $E^{\prime}$ and $E$ are isomorphic over $F$ but not over $\mathbb{Q}$. Since $C_{p} \subseteq E(F)[p]=E(\mathbb{Q})[p] \oplus E^{\prime}(\mathbb{Q})[p]$ and $E(\mathbb{Q})[p]=O$ it follows that $C_{p} \subseteq E^{\prime}(\mathbb{Q})[p]$. Obviously equality must hold, because of Mazur's theorem.

Lemma 3.4. Let $K$ be a number field such that $[K: \mathbb{Q}]$ is odd, $m \neq 2$ an integer and $E / K$ an elliptic curve with $j(E) \in \mathbb{Q}$ such that $C_{m} \subseteq E(K)$. If $E^{\prime} / \mathbb{Q}$ is an elliptic such that $j(E)=j\left(E^{\prime}\right)$ and $\mathbb{Q}\left(E^{\prime}[m]\right) \cap K=\mathbb{Q}$, then $G_{E^{\prime}}(m)$ is conjugate to a subgroup of $B$, where

$$
B:=\left\{\left[\begin{array}{cr} 
\pm 1 & * \\
0 & *
\end{array}\right]\right\} \subseteq \mathrm{GL}_{2}(\mathbb{Z} / m \mathbb{Z}) .
$$

Proof. By [37, Corollary 5.25.] we see that $\operatorname{Gal}\left(K \mathbb{Q}\left(E^{\prime}[m]\right) / K\right) \leq B$ (up to conjugacy). Since $\mathbb{Q}\left(E^{\prime}[m]\right) \cap K=\mathbb{Q}$ we have $\operatorname{Gal}\left(K \mathbb{Q}\left(E^{\prime}[m]\right) / K\right) \cong \operatorname{Gal}(\mathbb{Q}(E[m]) / \mathbb{Q})$ by a basic Galois theory argument. Therefore, $G_{E^{\prime}}(m)$ is conjugate to a subgroup of $B$.

Lemma 3.5. Let $K$ be a number field such that $[K: \mathbb{Q}] \geq 5$ is prime and $E / K$ be an elliptic curve with rational j-invariant and assume that $C_{2^{k} \cdot 3^{l}} \subseteq E(K)$. Then $E^{\prime}$ has a rational $2^{k} \cdot 3^{l}$-isogeny.

Proof. By [2, Corollary 2.8.] we have $\left|\mathrm{GL}_{2}\left(\mathbb{Z} / 2^{k} \mathbb{Z}\right)\right|=2^{4 k-3} \cdot 3$ and $\left|\mathrm{GL}_{2}\left(\mathbb{Z} / 3^{k} \mathbb{Z}\right)\right|=$ $3^{4 k-3} \cdot 2^{4}$. Let $p \in\{2,3\}$ be a prime number. Since $G_{E}(p) \leq \mathrm{GL}_{2}\left(\mathbb{Z} / p^{k} \mathbb{Z}\right)$, it follows that $[\mathbb{Q}(E[p]): \mathbb{Q}]=\left|G_{E}(p)\right|$ divides $\left|\mathrm{GL}_{2}\left(\mathbb{Z} / p^{k} \mathbb{Z}\right)\right| \in\left\{2^{4 k-3} \cdot 3,3^{4 k-3} \cdot 2^{4}\right\}$ so $\mathbb{Q}(E[p])$ has a trivial intersection with $K$, i.e. $\mathbb{Q}(E[p]) \cap K=\mathbb{Q}$. The claim now follows from Lemma 3.4 .

Lemma 3.6. Let $K$ be a number field such that $[K: \mathbb{Q}] \neq 5$ is an odd prime and $E / K$ be an elliptic curve with rational $j$-invariant such that $C_{5} \subseteq E(K)_{\text {tors }}$. Then $E$ is a base change of an elliptic curve defined over $\mathbb{Q}$. If $[K: \mathbb{Q}]=5$ and $C_{5} \subseteq E(K)_{\text {tors }}$, then $E^{\prime}$ has a rational 5-isogeny.

Proof. Assume that $[K: \mathbb{Q}] \neq 5$ is an odd prime and $E$ is not a base change of an elliptic curve defined over $\mathbb{Q}$. Since $C_{5} \subseteq E(K)$, it follows that $C_{5} \subseteq E^{\prime}(L)$. Let $P_{5}$ be a point of order 5 on $E^{\prime}(L)$. We have that $\mathbb{Q}\left(P_{5}\right) \subseteq L$ and so $\left[\mathbb{Q}\left(P_{5}\right): \mathbb{Q}\right]$ divides $[L: \mathbb{Q}]=2 p$, where $p=[K: \mathbb{Q}]$. By [13, Table 1], we see that the only possibilities for $\left[\mathbb{Q}\left(P_{5}\right): \mathbb{Q}\right]$ are 1 and 2 . Now we apply Lemma 3.3 to $E^{\prime}$ to obtain a quadratic twist $E^{\prime \prime} / \mathbb{Q}$ such that $C_{5} \subseteq E^{\prime \prime}(\mathbb{Q})$. Since $E$ and $E^{\prime \prime}$ are quadratic twists, they are isomorphic over some quadratic extension $L^{\prime}$ of $K$ and we have $C_{5} \oplus C_{5} \subseteq E(K)[5] \oplus E^{\prime \prime}(K)[5] \cong E^{\prime \prime}\left(L^{\prime}\right)[5]$. The Weil pairing implies that $\mathbb{Q}\left(\zeta_{5}\right) \subseteq L^{\prime}$ and so $\left[\mathbb{Q}\left(\zeta_{5}\right): \mathbb{Q}\right]=4$ divides $\left[L^{\prime}: \mathbb{Q}\right]=2 p$, which is impossible. 
If $[K: \mathbb{Q}]=5$, by applying the same reasoning as in the previous paragraph it can be easily seen that $E^{\prime}$ must have a rational 5-isogeny.

Lemma 3.7. Let $K$ be a number field such that $[K: \mathbb{Q}] \neq 3,7$ is prime and $E / K$ be an elliptic curve with rational $j$-invariant such that $C_{7} \subseteq E(K)_{\text {tors }}$. Then $E$ is a base change of elliptic curve defined over $\mathbb{Q}$. If $[K: \mathbb{Q}]=7$ and $C_{7} \subseteq E(K)_{\text {tors }}$, then $E^{\prime}$ has a rational 7-isogeny. If $[K: \mathbb{Q}]=3$ and $C_{7} \subseteq E(K)_{\text {tors }}$, then $E^{\prime}$ has a rational 7-isogeny unless $E^{\prime}$ has LMFDB label 2450.y1 or 2450.z1 (or equivalently, if $G_{E^{\prime}}(7)$ is conjugate to a group with label $7 N s .2 .1$.).

Proof. The proof is the same as in Lemma 3.6 .

We will classify torsion growth of $E^{\prime}$, if $E^{\prime}$ has LMFDB label 2450.y1 or 2450.z1 separately.

Lemma 3.8. Let $E^{\prime} / \mathbb{Q}$ be a curve with LMFDB label $2450 . \mathrm{y} 1$ or $2450 . \mathrm{z1}$ and let $L$ be a number field such that $[L: \mathbb{Q}]=2 p$, where $p$ is prime. Then $E^{\prime}(L)_{\text {tors }} \in$ $\left\{C_{1}, C_{2}, C_{2} \oplus C_{2}, C_{7}\right\}$.

Proof. Let $q \neq 7$ be a prime and let $E^{\prime}$ be any of these two curves. Then $G_{E^{\prime}}(q)=$ $\mathrm{GL}_{2}(\mathbb{Z} / q \mathbb{Z})$. Therefore, a point $P_{q}$ of order $q$ on $E^{\prime}$ satisfies $\left[\mathbb{Q}\left(P_{q}\right): \mathbb{Q}\right]=q^{2}-1$. If $P_{q} \in E^{\prime}(L)$, then $\left[\mathbb{Q}\left(P_{q}\right): \mathbb{Q}\right]=q^{2}-1$ would divide $[L: \mathbb{Q}]=2 p$. This implies that $q=2$ and $p=3$.

Consider the case $q=7$. If $P_{7} \in E^{\prime}(L)$ is a point of order 7 , by [13, Table 1] we have $[L: \mathbb{Q}]=6$. Using the algorithm from [12] we see that if $C_{7} \subseteq E^{\prime}(L)$, where $[L: \mathbb{Q}]=12$, then $C_{7}=E^{\prime}(K)_{\text {tors }}$.

From now on, assume that $E^{\prime}$ is not one of these two curves, i.e. if $C_{7} \subseteq E(K)$, then $E^{\prime}$ will have a rational 7-isogeny by Lemma 3.7 .

Lemma 3.9. Let $K$ be a number field such that $[K: \mathbb{Q}]$ is odd. Then there does not exist an elliptic curve $E / K$ with rational $j$-invariant such that $C_{16} \subseteq E(K)$.

Proof. Assume the contrary, that $C_{16} \subseteq E(K)$. It follows that $C_{16} \subseteq E^{\prime}(L)$ and let $P_{16}$ be a point of order 16 in $E^{\prime}(L)$. Since $\left[\mathbb{Q}\left(P_{16}\right): \mathbb{Q}\right]$ divides $[L: \mathbb{Q}]$ and 4 does not divide $[L: \mathbb{Q}]$, we have that $\left[\mathbb{Q}\left(P_{16}\right): \mathbb{Q}\right]$ is not divisible by 4 . By the results of $\left[11\right.$, we see that $G_{E^{\prime}}(16) \in\left\{H_{235 l}, H_{235 m}\right\}$ and $\left[\mathbb{Q}\left(P_{16}\right): \mathbb{Q}\right]=2$. In both cases we have $\left|G_{E^{\prime}}(16)\right|=256$. By Lemma 3.4. up to conjugacy we have $G_{E^{\prime}}(16) \leq B$ (where $B$ is a subgroup of upper triangular matrices defined in Lemma 3.4). Since $|B|=256$, the equality holds. But $-I \notin G_{E}(16)$ and $-I \in B$, a contradiction.

Lemma 3.10. Let $K$ be a number field such that $[K: \mathbb{Q}]$ is odd and let $E / K$ be an elliptic curve with rational j-invariant. Then $E(K)$ can't contain $C_{2} \oplus C_{12}$.

Proof. Assume that $C_{2} \oplus C_{12} \subseteq E(K)$. This implies $C_{2} \oplus C_{12} \subseteq E^{\prime}(L)$ and $[L: \mathbb{Q}]$ is not divisible by 4 . By [13, Table 1] we see that $E^{\prime}$ has a rational 3-isogeny and denote by $\left\langle P_{3}\right\rangle$ the kernel of such isogeny. Obviously $G_{E^{\prime}}(4)$ is not surjective, because otherwise a point $P_{4}$ of order 4 on $E^{\prime}$ would be defined over degree 12 extension of $\mathbb{Q}$, so 12 would divide $[L: \mathbb{Q}]$. By $\left[32\right.$, Theorem], we have that $G_{E^{\prime}}(4) \subseteq$ $H_{i}$, where $i \in\{9,10,11,12,13\}$. Since $\left|H_{i}\right|=16$, for $i \in\{9,10,11,12,13\}$ it follows that $\left[\mathbb{Q}\left(E^{\prime}[4]\right): \mathbb{Q}\right]=\left|G_{E^{\prime}}(4)\right|$ is a power of 2 . This implies that $\mathbb{Q}\left(E^{\prime}[4]\right) \cap K=\mathbb{Q}$. Since having a 2-torsion is twist invariant property and $E(K)[2]=C_{2} \oplus C_{2}$, we have $E^{\prime}(K)[2]=C_{2} \oplus C_{2}$. We now see that $\mathbb{Q}\left(E^{\prime}[2]\right) \subseteq \mathbb{Q}\left(E^{\prime}[4]\right) \cap K=\mathbb{Q}$, so $\mathbb{Q}(E[2])=\mathbb{Q}$. Since $C_{4} \subseteq E(K)$, by lemma 3.4 $E^{\prime}$ has a rational 4-isogeny. Let $P_{4}$ 
be a kernel of that isogeny and $Q_{2}$ be such that $\left\{2 P_{4}, Q_{2}\right\}$ is a basis for $E[2]$. The subroups $\left\langle P_{3}+P_{4}\right\rangle$ and $\left\langle Q_{2}\right\rangle$ are kernels of independent 12 and 2-isogenies, so $E^{\prime}$ is isogenous over $\mathbb{Q}$ to a curve $E^{\prime \prime} / \mathbb{Q}$ with a rational 24-isogeny, which is impossible by Theorem 2.3

Lemma 3.11. Let $[K: \mathbb{Q}]=p$ be a prime and let $E / K$ be an elliptic curve with rational j-invariant. Then $E(K)$ can't contain $C_{27}$.

Proof. If $p \geq 5$, by Lemma 3.5, $E^{\prime}$ has a rational 27-isogeny, so it has CM and so $E$ has $\mathrm{CM}$ as well, which contradicts our assumption. On the other hand, if $p=3$ then $E^{\prime}(L)$ would contain $C_{27}$, so $C_{27} \in \Phi_{\mathbb{Q}}(6)$, which isn't true by Theorem 2.8 .

Lemma 3.12. Let $[K: \mathbb{Q}]=p \geq 5$ be a prime and let $E / K$ be an elliptic curve with rational $j$-invariant. Then $E(K)$ can't contain $C_{18}$.

Proof. Since $E^{\prime}$ has a rational 2-isogeny, it has a rational point $P_{2}$ of order 2 . Let $P_{9} \in E^{\prime}(L)$ be a point of order 9 . We have that $\left[\mathbb{Q}\left(P_{9}\right): \mathbb{Q}\right]$ divides $[L: \mathbb{Q}]=2 p$. On the other hand, by $\left[13\right.$, Proposition 4.6.] we have that $\left[\mathbb{Q}\left(P_{9}\right): \mathbb{Q}\left(3 P_{9}\right)\right]\left[\mathbb{Q}\left(3 P_{9}\right): \mathbb{Q}\right]$ divides $18^{2}$, and since $\operatorname{gcd}\left(18^{2}, 2 p\right)=2$, we have $\left[\mathbb{Q}\left(P_{9}\right): \mathbb{Q}\right] \in\{1,2\}$. We conclude that $P_{9}$ is defined over an at most quadratic extension of $\mathbb{Q}$ and since $P_{2} \in E(\mathbb{Q})$, the point $P_{2}+P_{9}$ of order 18 on $E$ is defined over a quadratic number field, which is imossible since $C_{18} \notin \Phi_{\mathbb{Q}}(2)$, by Theorem 2.4

$$
\text { 4. }[K: \mathbb{Q}]=p, p \geq 11
$$

In this subsection, let $K$ denote a number field such that $[K: \mathbb{Q}]=p$, where $p \geq 11$ is a prime number.

If $P_{n} \in E(K)$ is a point of order $n$ then $C_{n} \subseteq E^{\prime}(L)$. By Lemma 3.1 we only need to consider those integers $n$ whose prime factors are contained in $R_{\mathbb{Q}}(2 p)=\{2,3,5,7\}$.

Theorem 4.1. Let $K$ be a number field such that $[K: \mathbb{Q}]=p \geq 11$ is prime and let $E / K$ be an elliptic curve with rational $j$-invariant. If $E(K)$ contains a point of order $n>1$, then $C_{n} \in \Phi(1)$.

Proof. Assume that $E(K)$ contains a point $P_{n}$ of order $n$. By Lemma 3.1 prime factors of $n$ are contained in $\{2,3,5,7\}$. Therefore, write $n=2^{a} 3^{b} 5^{c} 7^{d}$, where $a, b, c, d \geq 0$. If $(c, d) \neq(0,0)$, then by Lemma 3.6 and Lemma 3.7 we have that $E$ is a base change of an elliptic curve defined over $\mathbb{Q}$, so the claim holds by [13, Corollary 7.3.]. Consider now the case when $c=d=0$. By Lemma 3.5, $E^{\prime}$ has a rational $2^{a} 3^{b}$-isogeny. By Theorem 2.3 we have

$$
n=2^{a} 3^{b} \in\{1,2,3,4,6,8,9,12,16,18,27\} .
$$

Among these values of $n$, we have $C_{n} \notin \Phi(1)$ only for $n \in\{16,18,27\}$. But each of these cases is impossible by Lemma 3.9, Lemma 3.12 and Lemma 3.11.

$$
\text { 5. }[K: \mathbb{Q}]=7
$$

In this subsection, let $K$ denote a number field such that $[K: \mathbb{Q}]=7$.

If $P_{n} \in E(K)$ is a point of order $n$ then $C_{n} \subseteq E^{\prime}(L)$. By Lemma 3.1 we only need to consider those integers $n$ whose prime factors are contained in $R_{\mathbb{Q}}(14)=\{2,3,5,7\}$.

Lemma 5.1. Let $E / K$ be an elliptic curve with rational j-invariant. Then $E(K)$ can't contain $C_{49}, C_{21}$ or $C_{14}$. 
Proof. $C_{49}$ : This is proven in [12, Lemma 2.8.].

$C_{21}$ : By Lemma 3.5 and Lemma $3.7 E^{\prime}$ has a rational 3 and 7 -isogenies, so it has a rational 21-isogeny so $j(E) \in\left\{-3^{2} \cdot 5^{6} / 2^{3}, 3^{3} \cdot 5^{3} / 2,3^{3} \cdot 5^{3} \cdot 101^{3} / 2^{21},-3^{3} \cdot 5^{3} \cdot 383^{3} / 2^{7}\right\}$. Using the division polynomial method in Magma [1, we see that this is impossible.

$C_{14}$ : By Lemma 3.5 and Lemma $3.7 E^{\prime}$ has a rational 2 and 7 -isogenies, so it has a rational 14 -isogeny so $E^{\prime}$ has $\mathrm{CM}$ which implies that $E$ has $\mathrm{CM}$ aswell.

Theorem 5.2. Let $K$ be a number field such that $[K: \mathbb{Q}]=7$. If $E(K)$ contains a point of order $n>1$, then $C_{n} \in \Phi(1)$.

Proof. Assume that $E(K)$ contains a point $P_{n}$ of order $n$. By Lemma 3.1, the prime factors of $n$ can only be $2,3,5,7$. Therefore, write $n=2^{a} 3^{b} 5^{c} 7^{d}$, where $a, b, c, d \geq 0$. If $c \neq 0$, then by Lemma 3.6 $E$ is a base change of elliptic curve defined over $\mathbb{Q}$, so $C_{n} \in \Phi_{\mathbb{Q}}(7)=\Phi(1)$. Assume that $c=0$. If $d \neq 0$, Lemma 5.1 implies that $a=b=0$, so $C_{n}=C_{7} \in \Phi(1)$. It remains to consider the case $c=d=0$. By Lemma 3.5. $E^{\prime}$ has a rational $2^{a} 3^{b}$-isogeny. By Theorem 2.3 we have

$$
2^{a} 3^{b} \in\{1,2,3,4,6,8,9,12,16,18,27\} .
$$

Among these values of $n$, we have $C_{n} \notin \Phi(1)$ only for $n \in\{16,18,27\}$. But each of these cases is impossible by Lemma 3.9, Lemma 3.12 and Lemma 3.11.

$$
\text { 6. }[K: \mathbb{Q}]=5
$$

In this subsection, let $K$ denote a number field such that $[K: \mathbb{Q}]=5$.

If $P_{n} \in E(K)$ is a point of order $n$ then $C_{n} \subseteq E^{\prime}(L)$. By Lemma 3.1 we only need to consider those integers $n$ whose prime factors are contained in $R_{\mathbb{Q}}(10)=$ $\{2,3,5,7,11\}$.

Lemma 6.1. Let $E / K$ be an elliptic curve with rational $j$-invariant. Then $E(K)$ can't contain $C_{121}, C_{15}, C_{50}$ or $C_{125}$.

Proof. $C_{121}$ : This is proven in [12, Lemma 2.9.].

$C_{15}$ : By Lemma 3.5 and Lemma 3.6, $E^{\prime}$ has a rational 15-isogeny, so $j\left(E^{\prime}\right) \in$ $\left\{-5^{2} / 2,-5^{2} \cdot 241^{3} / 2^{3},-5 \cdot 29^{3} / 2^{5}, 5 \cdot 211^{3} / 2^{15}\right\}$. Let $\left\langle P_{15}\right\rangle$ be the kernel of such a rational 15 -isogeny and $P_{15}$ a point of order 15 on $E^{\prime}$. Since $\operatorname{Gal}(\mathbb{Q}(E[15]) / \mathbb{Q})$ acts on $\left\langle P_{15}\right\rangle$, we get that $\left[\mathbb{Q}\left(P_{15}\right): \mathbb{Q}\right]$ divides $\left|(\mathbb{Z} / 15 \mathbb{Z})^{\times}\right|=8$. On the other hand, $\mathbb{Q}\left(P_{15}\right) \subseteq L$ and since $[L: \mathbb{Q}]=10$, we have $\left[\mathbb{Q}\left(P_{15}\right): \mathbb{Q}\right]=2$. By [33, Theorem 2.c)], the LMFDB label of $E^{\prime}$ is 50.b3, 50.b4, 50.a2 or 450.g4. Let $E^{\prime} \in\{50 . \mathrm{b3} 50 . \mathrm{b} 4\}$. Then we have $E^{\prime}(\mathbb{Q})[5]=C_{5}$ and since $C_{5} \oplus C_{5} \subseteq E(K)[5] \oplus E^{\prime}(K)[5] \cong E^{\prime}(L)[5]$, the Weil pairing implies that $\mathbb{Q}\left(\zeta_{5}\right) \subseteq L$ which is impossible. Assume now that $E^{\prime} \in\{50 . \mathrm{a} 2,450 . \mathrm{g} 4\}$. In these cases we have that $E^{\prime}(\mathbb{Q})[3]=C_{3}$. Applying the same reasoning as before, we conclude that $\mathbb{Q}\left(\zeta_{3}\right) \subseteq L$. On the other hand these two curves attain 15 -torsion point over $\mathbb{Q}(\sqrt{5})$ and $\mathbb{Q}(\sqrt{-15})$, respectively. Therefore we have $\mathbb{Q}(\sqrt{5}) \subseteq L$ or $\mathbb{Q}(\sqrt{-15}) \subseteq L$, but this is impossible since $\mathbb{Q}\left(\zeta_{3}\right) \subseteq L$ is a unique quadratic subextension of $L$, because $[L: \mathbb{Q}]=10$.

$C_{50}$ : By Lemma 3.5 we have that $E^{\prime}$ has rational 2 and 5-isogenies. If $G_{E^{\prime}}(5) \subseteq$ $C_{s}(5)$, then $E^{\prime}$ has two independent rational 5-isogenies. Denote by $\langle P\rangle$ and $\langle Q\rangle$ 
the kernels of these isogenies. We have that $\left\langle P_{2}+P\right\rangle$ and $\langle Q\rangle$ are kernels of independent 10 and 5-isogenies, so $E^{\prime}$ is isogenous over $\mathbb{Q}$ to a curve $E^{\prime \prime} / \mathbb{Q}$ with a rational 50-isogeny, which is impossible by Theorem 2.3. Therefore we conclude that $G_{E^{\prime}}(5) \in\{5 B .1 .1,5 B .1 .2,5 B .1 .3,5 B .1 .4,5 B .4 .1,5 B .4 .2,5 B\}$. A calculation in Magma shows that if $E^{\prime}$ obtains a point $P_{25}$ of order 25 over a degree 5 or 10 extension of $\mathbb{Q}$, then it must have a rational 25-isogeny. But since it has a rational 2 -isogeny as well, it must have a rational 50 -isogeny, which is impossible by Theorem 2.3 .

$C_{125}$ : This is proven in [12, Proposition 2.7.].

Theorem 6.2. Let $K$ be a number field such that $[K: \mathbb{Q}]=5$. If $E(K)$ contains a point of order $n>1$, then $C_{n} \in \Phi_{\mathbb{Q}}(5)$.

Proof. Assume that $E(K)$ contains a point $P_{n}$ of order $n$. By Lemma 3.1, the prime factors of $n$ can only be $2,3,5,7,11$. Therefore, write $n=2^{a} 3^{b} 5^{c} 7^{d} 11^{e}$, where $a, b, c, d, e \geq 0$. If $d \neq 0$, then by Lemma $3.7 E$ is a base change of an elliptic curve defined over $\mathbb{Q}$, so $C_{n} \in \Phi_{\mathbb{Q}}(5)$. Assume that $d=0$. If $e \neq 0$, from [13, Table 2] we see that $E^{\prime}$ has a rational 11-isogeny. If one of $a, b, c$ is not zero, $E^{\prime}$ would have a rational $p$-isogeny, where $p \in\{2,3,5\}$ so it would have a rational $11 p$-isogeny which is impossible by Theorem 2.3. Therefore we have $a=b=c=0$. If $e \geq 2$, this is impossible by Lemma 6.1. We conclude that if $e \geq 1$, then $n=11$. Consider now the case when $d=e=0$. If $c \geq 1$, by Lemma 3.5 and Lemma $3.6 E^{\prime}$ has a rational $2^{a} 3^{b} 5$-isogeny. By Theorem 2.3 we have $2^{a} 3^{b} 5 \in\{5,10,15\}$, so $(a, b) \in\{(0,0),(1,0),(0,1)\}$ and $n \in\left\{5^{c}, 2 \cdot 5^{c}, 3 \cdot 5^{c}\right\}$. We have that this is impossible by Lemma 6.1 unless $n \in\{5,10,25\}$, but for those values of $n$ we have $C_{n} \in \Phi_{\mathbb{Q}}(5)$. Finally let us consider the case when $n=2^{a} 3^{b}$. By Theorem 2.3 we know that

$$
n=2^{a} 3^{b} \in\{1,2,3,4,6,8,9,12,16,18,27\} .
$$

Removing the $n$ for which $C_{n} \in \Phi_{\mathbb{Q}}(5)$ we only need to consider $n \in\{16,18,27\}$. These three cases have been proven to be impossible in Lemma 3.9. Lemma 3.11 and Lemma 3.12 .

Lemma 6.3. Let $E / K$ be an elliptic curve with rational $j$-invariant. Then $E(K)$ can't contain $C_{2} \oplus C_{10}$ or $C_{2} \oplus C_{12}$.

Proof. $C_{2} \oplus C_{12}$ : This has been proven in Lemma 3.10

$C_{2} \oplus C_{10}$ : Since 2-torsion does not change when twisting, $E(K)[2]=C_{2} \oplus C_{2}$ implies that $E^{\prime}(K)[2]=C_{2} \oplus C_{2}$. From $[K: \mathbb{Q}]=5$ it follows that $E^{\prime}(\mathbb{Q})[2]=C_{2} \oplus C_{2}$. Lemma 3.6 implies that $E^{\prime}$ has a rational 5-isogeny and we know that it has full 2torsion over $\mathbb{Q}$. It follows that $E^{\prime}$ is isogenous over $\mathbb{Q}$ to $E^{\prime \prime} / \mathbb{Q}$ that has 20 -isogeny, which is impossible by Theorem 2.3 .

$$
\text { 7. }[K: \mathbb{Q}]=3
$$

In this subsection, let $K$ denote a number field such that $[K: \mathbb{Q}]=3$. If $P_{n} \in E(K)$ is a point of order $n$ then $C_{n} \subseteq E^{\prime}(L)$. By Lemma 3.1 we only need to consider those integers $n$ whose prime factors are contained in $R_{\mathbb{Q}}(6)=$ $\{2,3,5,7,13\}$. 
Theorem 7.1. Let $K$ be a number field such that $[K: \mathbb{Q}]=3$. If $E(K)$ contains a point of order $n>1$, then $C_{n} \in \Phi_{\mathbb{Q}}(3)$.

Proof. Let $C_{n} \subseteq E(K)$. We have that $C_{n} \subseteq E^{\prime}(L)$ where $[L: \mathbb{Q}]=6$. Therefore, by Theorem 2.8 we have that $C_{n}$ is equal to the one of the following groups:

$$
C_{m}: m=1, \ldots, 10,12,13,14,15,16,18,21,30 .
$$

If $C_{n} \in \Phi_{\mathbb{Q}}(3)$, we are done. Assume that this isn't the case. Then $n \in\{15,16,30\}$. Obviously it's enough to show that $n=15$ and $n=16$ is impossible. If $n=15$, by Lemma 3.6 we have that $E$ is a base change of an elliptic curve defined over $\mathbb{Q}$. Since $C_{15} \notin \Phi_{\mathbb{Q}}(3)$, we are done. The case when $n=16$ has been proven in Lemma 3.9 .

Lemma 7.2. Let $E / K$ be an elliptic curve with rational j-invariant. Then $E(K)$ can't contain $C_{2} \oplus C_{10}, C_{2} \oplus C_{12}$ or $C_{2} \oplus C_{18}$.

Proof. $C_{2} \oplus C_{10}$ : If $C_{2} \oplus C_{10} \subseteq E(K)$, by Lemma 3.6 and Lemma 3.7 we have that $E$ is a base change of an elliptic curve defined over $\mathbb{Q}$, so $C_{2} \oplus C_{10} \in \Phi_{\mathbb{Q}}(3)$, which contradicts Theorem 2.5.

$C_{2} \oplus C_{12}$ : This has been proven in Lemma 3.10 .

$C_{2} \oplus C_{18}$ : Assume that $C_{2} \oplus C_{18} \subseteq E(K)$. A point $P_{3}$ of order 3 on $E^{\prime}$ is defined over an at most quadratic extension of $\mathbb{Q}$, by [12, Table 1]. Using Lemma 3.3 we can assume that $E^{\prime}(\mathbb{Q})[3]=C_{3}$. Since $C_{3} \oplus C_{3}=E(K)[3] \oplus E^{\prime}(K)[3] \cong E^{\prime}(L)[3]$, $C_{9} \subseteq E^{\prime}(L)$ and $E^{\prime}(L)[2]=C_{2} \oplus C_{2}$, it follows that $C_{6} \oplus C_{18} \subseteq E^{\prime}(L)$. Obviously $G_{E}(2) \neq 2 B$. By Theorem 2.8, this is impossible.

$$
\text { 8. }[K: \mathbb{Q}]=2
$$

In this subsection, let $K$ denote a number field such that $[K: \mathbb{Q}]=2$.

Theorem 8.1. Let $K$ be a number field such that $[K: \mathbb{Q}]=2$. If $E(K)$ contains a point of order $n>1$, then $C_{n} \in \Phi_{\mathbb{Q}}(2) \cup\left\{C_{13}\right\}$.

Proof. Obviously we have $\Phi_{\mathbb{Q}}(2) \subseteq \Phi_{j \in \mathbb{Q}}(2) \subseteq \Phi(2)$. By Theorem 2.2 and Theorem 2.4 we have $\Phi(2) \backslash \Phi_{\mathbb{Q}}(2)=\{11,13,14,18\}$. Let $n$ be one of those values. Obviously $E / K$ such that $E(K)$ contains a point $P_{n}$ of order $n$ can't be a base change of an elliptic curve defined over $\mathbb{Q}$, by Theorem 2.4. Therefore, let $E^{\prime} / \mathbb{Q}$ be an elliptic curve such that $j(E)=j\left(E^{\prime}\right)$ and let $L$ be a quartic field over which $E$ and $E^{\prime}$ are isomorphic. This implies that $E^{\prime}$ has a point of order $n \in\{11,14,18\}$, but this is impossible since $C_{n} \notin \Phi_{\mathbb{Q}}(4)$ by Theorem 2.6.

Let now $E^{\prime \prime} / \mathbb{Q}$ be an elliptic curve and $L$ a quartic Galois extension of $\mathbb{Q}$ such that $P_{13} \in E^{\prime \prime}(L)$, where $P_{13}$ is a point of order 13. Such an elliptic curve $E^{\prime \prime}$ and a field $L$ exist by [4, Theorem 1.2]. Denote by $K$ the intermediate field $\mathbb{Q} \subseteq K \subseteq L$. Since $L=K(\sqrt{d})$, consider a twist $E^{d} / K$ of $E^{\prime \prime} / K$ by $d$. We have

$$
C_{13} \cong E^{\prime \prime}(L)[13]=E^{d}(K)[13] \cong E^{\prime \prime}(K)[13]
$$

We conclude that $C_{13} \subseteq E^{d}(K)[13]$ and so $E^{d} / K$ is an elliptic curve with $j\left(E^{d}\right) \in \mathbb{Q}$ defined over a quadratic extension of $\mathbb{Q}$ with a point of order 13 . 
Remark: The sets $\Phi_{j \in \mathbb{Q}}(d)$ for $d=2,3$ were also determined by Manolis Tzortzakis in his thesis under the assumption that we know the set $\Phi(3)$. We did not assume the knowledge of $\Phi(3)$. The author has been working on determining the set $\Phi_{j \in \mathbb{Q}}(4)$ and has obtained partial results.

\section{ACKNOWLEDGEMENTS}

The author would like to thank his advisor Filip Najman for introducing him to this problem, for many helpful discussions and for help during the writing of this paper.

\section{REFERENCES}

[1] Wieb Bosma, John Cannon, and Catherine Playoust, The Magma algebra system. I. The user language, J. Symbolic Comput., 24 (1997), 235265.

[2] A.Bourdon and P. Pollack, Torsion subgroups of CM elliptic curves over odd degree number fields, Int. Math. Res. Not. IMRN (2017), no. 16, 4923-4961.

[3] A.Bourdon, P.L.Clark, Torsion points on CM elliptic curves over real number fields, Trans. Amer. Math. Soc. 369 (2017), no. 12, 8457-8496.

[4] M. Chou, Torsion of rational elliptic curves over quartic Galois number fields, J. Number Theory 160 (2016), 603-628.

[5] P. L. Clark, P. Corn, A. Rice and J. Stankiewicz, Computation on elliptic curves with comples multiplication, LMS J. Comput. Math. 17 (2014), 509-539.

[6] H. B. Daniels and E. González-Jiménez, On the torsion of rational elliptic curves over sextic fields, To appear in Mathematics of Computation data, arXiv: 1808.02887

[7] H. B. Daniels, A. Lozano-Robledo, F.Najman and A.V. Sutherland, Torsion subgroups of rational elliptic curves over the compositum of all cubic fields, Math. Comp. 87 (2018), 425458.

[8] M. Derickx and A. V. Sutherland, Torsion subgroups of elliptic curves over quintic and sextic number fields, Proc. Amer. Math. Soc. 145 (2017), 4233-4245.

[9] E. González-Jiménez, Complete classification of the torsion structures of rational elliptic curves over quintic number fields, Journal of Algebra 478 (2017) 484505.

[10] E. González-Jiménez, Compe classification of the torsion structures of rational elliptic curves over quintic number fields, J. Algebra 478 (2017), 484-505.

[11] E. González-Jiménez and A. Lozano-Robledo, On the minimal degree of definition of $p$ primary torsion subgroups of elliptic curves, Mathematical Research ter 24 (2017) 1067-1096.

[12] E. González-Jiménez and F. Najman, An Algorithm for determining torsion growth of elliptic curves, preprint.

[13] E. González-Jiménez and F. Najman and, Growth of torsion groups of elliptic curves upon base change, Math. Comp. to appear.

[14] E. González-Jiménez, F. Najman and J. M. Tornero, Torsion of rational elliptic curves over cubic fields, es over cubic fields, Rocky Mountain J. Math. 46 (2016), 1899-1917.

[15] T. Gužvić, Torsion growth of rational elliptic curves in sextic number fields, preprint.

[16] J. Han, The General Linear group over a ring, Bull. Korean Math. Soc. 43 (2006), No. 3, pp. 619-626.

[17] P. Ingram, Diophantine analysis and torsion points on elliptic curves, Proc. London Math. Soc. 94 (2007), 473-486.

[18] D. Jeon, C. H. Kim and A. Schweizer, On the torsion of elliptic curves over cubic number fields, Acta Arith. 113 (2004) 291-301.

[19] D. Jeon, C. H. Kim and E. Park, On the torsion of elliptic curves over quartic number fields, J. London Math. Soc. 74 (2006), 1-12.

[20] S. Kamienny, Torsion points on elliptic curves and q-coefficients of modular forms, Invent. Math. 109 (1992), 221-229.

[21] M. A. Kenku and F. Momose, Torsion points on elliptic curves defined over quadratic field, Nagoya Math. J. 109 (1988), 125-149.

[22] M. A. Kenku, The modular curve $X_{0}(39)$ and rational isogeny, Math. Proc. Cambridge Philos. Soc. 85 (1979), 21-23. 
[23] M. A. Kenku, The modular curves $X_{0}(65)$ and $X_{0}(91)$ and rational isogeny, Math. Proc. Cambridge Philos. Soc. 87 (1980), 15-20.

[24] M. A. Kenku, The modular curve $X_{0}(169)$ and rational isogeny, J. London Math. Soc. (2) 22 (1980), 239-244.

[25] M. A. Kenku, The modular curve $X_{0}(125), X_{1}(25)$ and $X_{1}(49)$, J. London Math. Soc. (2) 23 (1981), 415-427.

[26] LMFDB Collaboration, The L-functions and modular forms database, available at http://www.lmfdb.org.

[27] A. Lozano-Robledo and E. González-Jiménez, On the minimal degree of definition of pprimary torsion subgroups of elliptic curves, Math. Res. t. 24 (2017), 1067-1096. (data files http://matematicas.uam.es/ enrique.gonzalez.jimenez/).

[28] A. Lozano-Robledo, On the field of definition of p-torsion points on elliptic curves over the rationals, Math. Ann 357 (2013), 279-305.

[29] B. Mazur, Modular curves and the Eisenstein ideal, Inst. Hautes tudes Sci. Publ. Math. 47 (1978), 33-186.

[30] B. Mazur, Rational isogenies of prime degree, Invent. Math. 44 (1978), pp. 129-162.

[31] L. Merel, Bornes pour la torsion des courbes elliptiques sur les corps de nombres, Invent. Math. 124 (1996), 437-449.

[32] J. S. Morrow, Composite images of Galois for elliptic curves over $\mathbb{Q}$ and entanglement fields, Math. Comp. 88 (2019), 2389-2421.

[33] F. Najman, Torsion of rational elliptic curves over cubic fields and sporadic points on $X_{1}(n)$, Math. Res. ters, 23 (2016) 245-272.

[34] F. Najman, Complete classification of torsion of elliptic curves over quadratic cyclotomic fields, J. Number Theory 130 (2010), 1964-1968.

[35] L. D. Olson, Points of finite order on elliptic curves with complex multiplication, Manuscripta Math. 14 (1974) 195-205.

[36] J. Rouse and D. Zuerick-Brown Elliptic curves over $\mathbb{Q}$ and 2-adic images of Galois, Research in Number Theory 1:12, 2015. (Data files and subgroup descriptions available at http://users.wfu.edu/rouseja/2adic/).

[37] A. V. Sutherland, Computing images of Galois representations attached to elliptic curves, Forum Math. Sigma 4 (2016), e4, 79 pp.

[38] L. Washington, Elliptic Curves. Number Theory and Cryptography, Discrete Mathematics and its Applications (Boca Raton), Chapman and Hall/CRC, Boca Raton, 2003.

[39] D. Zywina, On the possible images of the mod l representations associated to elliptic curves over $\mathbb{Q}$, arXiv: 1508.07660 .

Department of Mathematics, Faculty of Science, University of Zagreb, Bijenička Cesta 30, 10000 Zagreb, Croatia

E-mail address: tguzvic@math.hr 